\title{
Functional evaluation of horse chestnut seed and its application in the production of compounded paper for effective utilization of an untapped resource
}

\author{
Tetsuya Takahashi $^{1} \cdot$ Yoko Tsurunaga $^{2} \cdot$ Werner F. Schmidt $^{3} \cdot$ Katsumi Yoshino $^{4}$
}

Received: 15 March 2017 / Accepted: 18 June 2017/Published online: 27 July 2017

(C) The Japan Wood Research Society 2017

\begin{abstract}
Many streets in Europe and the US are lined with horse chestnut trees. However, its fruit is inedible and poorly utilized. Therefore, its effective utilization has been a big issue. For recycling of horse chestnut seed, it was separated into "sarcocarp and endodermis" and "outer theca"; each part was finely ground for analysis of its functions. Ground horse chestnut seed showed excellent antibacterial activity toward Staphylococcus aureus, Escherichia coli, and Streptococcus mutans. Antibacterial activity was particularly strong against $E$. coli, which is a Gram-negative bacterium. The "outer theca" part showed a higher antibacterial activity than did the "sarcocarp and endodermis" part. It was found that the polyphenol content of the "outer theca" of the horse chestnut fruit was approximately three times that of the "sarcocarp and endodermis"; these polyphenols are believed to play a role in the seed's antioxidation capacity. The ground outer theca of horse chestnut seed showed oxygen radical absorbance capacity (ORAC) of $531 \mathrm{mmol}$ Trolox equivalent (TE)/g. Antioxidant activity was assumed to contribute to the antibacterial activity. In other words, antioxidant activity was assumed to damage the peptidoglycan layer of the bacterial cell wall. For possible
\end{abstract}

Tetsuya Takahashi

takahashi@hmn.shimane-u.ac.jp

1 Faculty of Human Sciences, Shimane University, 1060 Nishikawatsu-cho, Matsue, Shimane 690-8504, Japan

2 Faculty of Education, Shimane University, 1060 Nishikawatsu-cho, Matsue, Shimane 690-8504, Japan

3 Formerly Hahn-Meitner Institute, Dahler Weg 172, 14167 Berlin, Germany

4 Shimane Institute for Industrial Technology, 1, Hokuryo-cho, Matsue, Shimane 690-0816, Japan recycling of horse chestnut seed, compounded paper containing the ground outer theca blended with pulp fibers was prepared. In this study, compounded paper containing ground "outer theca" was prepared using a traditional Washi paper-making method. The compounded paper was found to retain high deodorizing activity of the ground theca. Furthermore, although mechanical properties deteriorated as the proportion of the ground outer theca increased, these parameters remained adequate for practical applications. The compounded paper may be expected to have a wide range of applications as a highly functional paper with high gas permeability, deodorant activity, and antibacterial activity.

Keywords Horse chestnut seed - Antibacterial activity · Deodorant ability - Oxygen radical absorbance capacity . Compounded papers

\section{Introduction}

Horse chestnut (marronnier) is a tall deciduous tree that belongs to genus Aesculus of family Hippocastanaceae $[1,2]$. It is a magnificent tree with loosely expanding big branches. Space created by horse chestnut trees lining a street has beneficial effects such as charm and the feeling of peace. This is why streets are lined with many horse chestnut trees in Europe. The US horse chestnut trees bear seed from September to November, and the fallen seed abundantly covers roadsides. The fallen seed is taken away by rodents for caching [3]. Nonetheless, the fallen seeds, which are quite large, remain concentrated under canopies of mother trees and need to be removed. The horse chestnut seed is covered with a hard theca. When the theca is cracked, a seed appears that closely resembles a chestnut 
seed. Horse chestnut seed contains a high concentration of saponin as well as aesclin and coumarin glycoside, which exert antithrombotic action and can be used as pharmaceutical drugs [4]. On the other hand, because horse chestnut seed is not edible, it is not much utilized and is treated as waste. Its effective utilization is a big issue at present. To effectively utilize horse chestnut seed, it is necessary to examine the properties of ground seed.

In this study, horse chestnut seed was separated into two parts: one consisting of the sarcocarp and endodermis and the other consisting of the outer theca. Various characteristics of each part were analyzed for the purpose of effective utilization. The above-mentioned experiment revealed high antibacterial activities of horse chestnut seed toward all the tested bacterial species. This activity was found to be particularly strong in the outer theca. In addition, an attempt was made to prepare compounded paper to take advantage of this product. The compounded paper was tested for its performance, which is described in this report.

\section{Materials and methods}

\section{Samples}

\section{Horse chestnut seed}

These seeds were sampled from horse chestnut trees (Scientific name: Aesculus hippocastanum L.) lining Dahlemer Strasse, in the Zehlendorf area of Berlin in Germany, in October 2013. A piece of seed was $3-4 \mathrm{~cm}$ in diameter (Fig. 1). Horse chestnut seed dried at room temperature was notched in the pericarp to separate the seed from pericarp (Fig. 1). The seed and pericarp were separately ground in an Oster blender (SUN BEAM OSTER) at $15,000 \mathrm{rpm}$ for approximately $2 \mathrm{~min}$, and then sifted through a 1-mm-mesh sieve to obtain particles approximately $1 \mathrm{~mm}$ in diameter. Because the sarcocarp and the endodermis firmly adhered to each other such that they could not be separated, the outer theca alone was peeled off to separate the "sarcocarp and endodermis" from the "outer theca" (Fig. 1).

\section{Testing for effects on bacteria}

Staphylococcus aureus (NBRC 12732), Escherichia coli (NBRC 3301), and Streptococcus mutans (NBRC 13955) were obtained from the National Institute of Technology and Evaluation, Independent Administrative Institute, and used to evaluate the sarcocarp and endodermis and the outer theca for antibacterial activities toward various bacteria. S. aureus is a Gram-positive coccus, E. coli is a
Gram-negative rod, and $S$. mutans is a Gram-positive, anaerobic streptococcus also known to cause dental caries.

\section{Preparation of compounded papers}

Compounded paper was prepared by the method reported previously [5-7]. Briefly, outer theca of horse chestnut seed was ground under wet conditions with a mass colloider (stone mill type crusher) with a clearance of $40 \mu \mathrm{m}$, before blending with desired amounts of pulp and latex binder. The pulp used was prepared by refining Canadian-bleached conifer kraft pulp to a standard freeness of $550 \mathrm{~mL}$ with a refiner. Aica Aibon RAX117 (Aica Kogyo, Kiyosu, Aichi, Japan), a latex binder (emulsion) made of styrene-butadiene rubber (SBR), was used to improve the adhesion between pulp and the ground outer theca of horse chestnut seed. Then, the ground outer theca of horse chestnut seed was blended with a prescribed amount of the bleached conifer kraft pulp and the latex binder. Compounded papers containing the ground outer theca of horse chestnut seed were prepared as described previously. The ground horse chestnut seed content was adjusted to $20,40,60 \mathrm{wt} \%$ in the formulation; the latex binder content was $0.3 \mathrm{wt} \%$ of the combined amount of the ground outer theca of horse chestnut seed and the pulp in all the formulations. Distilled water was added to the mixtures of the foregoing ratios, which were then stirred with a mixer for $10 \mathrm{~s}$ to obtain slurries of even dispersion. An angular-sheeting machine (PU-401; Tester Sangyo, Saitama, Japan) was used to adjust the slurries to a paper weight of $100 \mathrm{~g} / \mathrm{m}^{2}$. The sheets were pressed at $410 \mathrm{kPa}$ at room temperature and dried at $120{ }^{\circ} \mathrm{C}$ with a rotating drier to prepare compounded papers containing the ground outer theca of horse chestnut seed.

\section{Measurement method}

\section{Soluble polyphenol concentration}

Soluble polyphenol concentration was measured using the Folin method [8]. A test liquid was prepared by extracting $25 \mathrm{mg}$ of a ground part of horse chestnut seed with $1 \mathrm{~mL}$ of distilled water in a $1.5-\mathrm{mL}$ microtube (Violamo, AS ONE Corporation, Osaka, Japan) at $100{ }^{\circ} \mathrm{C}$ for $10 \mathrm{~min}$. Briefly, $90 \mu \mathrm{L}$ extract, $90 \mu \mathrm{L}$ Folin-Ciocalteu reagent, and $90 \mu \mathrm{L}$ $10 \%$ sodium carbonate were mixed in the well of a 96-well microplate (VIORAMO 96-well plate, Azone, Japan, Round-bottom type). After incubation for $60 \mathrm{~min}$ at room temperature, the reaction color was measured with a Sunrise-Thermo microplate reader (Tecan Inc., Salzburg, Austria) at $690 \mathrm{~nm}$. The quantity was expressed proportionally as $\mathrm{mg}(+)$-catechin per $100 \mathrm{~g}$ powder $(\mathrm{mg} / 100 \mathrm{~g}$ dry weight: "mg catechin eq./100 gDW") [8]. 

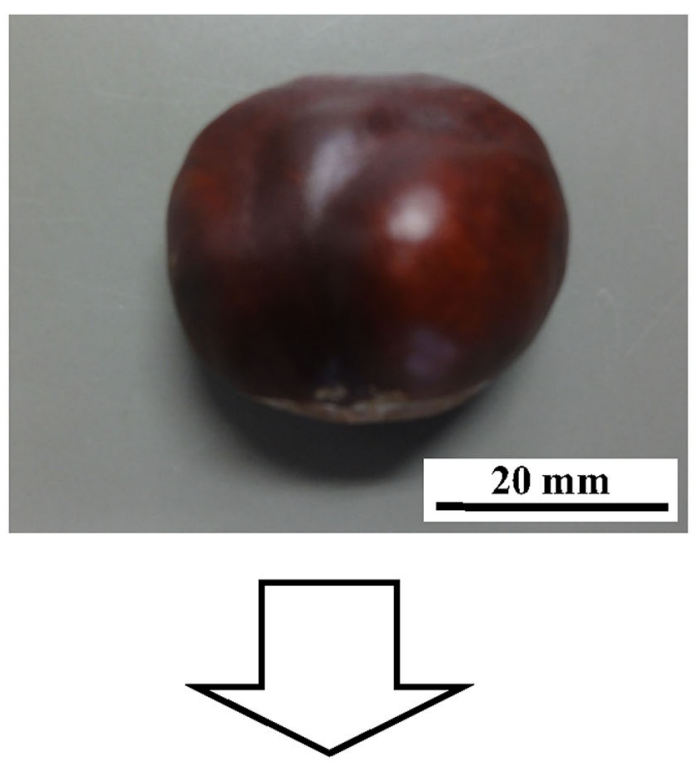

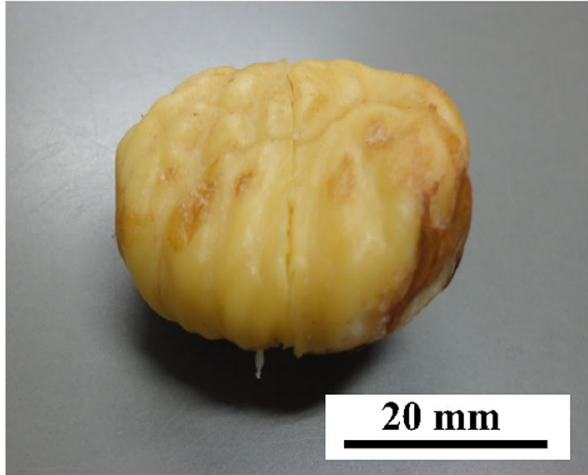

sarcocarp and endodermis

Fig. 1 Separation of parts of the horse chestnut seed by peeling

\section{Oxygen radical absorbance capacity (ORAC)}

A test liquid was prepared by extracting $25 \mathrm{mg}$ of a ground part of horse chestnut seed with $1 \mathrm{~mL}$ of distilled water in a 1.5-mL microtube (Violamo, AS ONE Corporation, Osaka, Japan) at $100{ }^{\circ} \mathrm{C}$ for $10 \mathrm{~min}$. A fluorescence plate reader (SH-9000Lab, CORONA ELECTRIC Co., Ltd., Ibaragi, Japan) kept at $37{ }^{\circ} \mathrm{C}$ was used to measure fluorescence intensity of fluorescein after we placed $35 \mu \mathrm{L}$ of a test liquid or a Trolox solution and then $115 \mu \mathrm{L}$ of a fluorescein solution (94.4 nM/75 mM-phosphate buffer solution, $\mathrm{pH}$ 7.4) in a well of a 96-well microplate (\#3072, Becton-Dickinson). Then, 8 min after addition of $50 \mu \mathrm{L}$ of an 2,2'-azobis-2-methyl-propanimidamide, dihydrochloride (AAPH) solution $(31.7 \mathrm{mM} / 75 \mathrm{mM}$-phosphate buffer solution, $\mathrm{pH}$ 7.4) and shaking, the microplate reader was used to take measurements at intervals of $2 \mathrm{~min}$ for $90 \mathrm{~min}$

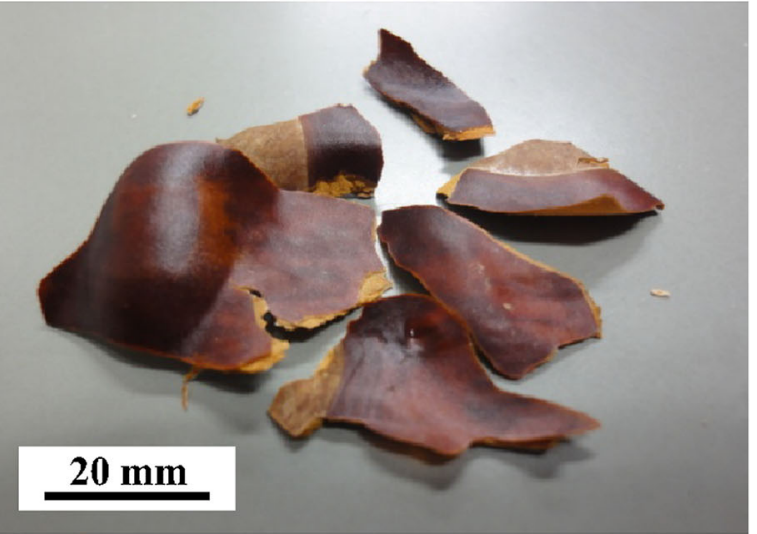

outer theca

to register the time course of fluorescence intensity. An excitation wavelength of $485 \mathrm{~nm}$ and a detection wavelength of $520 \mathrm{~nm}$ were used for measurement on the microplate reader. Hydroxyl Radical Antioxidant Capacity (H-ORAC) values were expressed in Trolox equivalents (mmol TE/g).

\section{Antibacterial properties}

With JIS-L-1902 as a Ref. [9], antibacterial activity assays were performed on the ground parts of horse chestnut seed and compounded paper. A sample weighing $0.04 \mathrm{~g}$ was placed in a $15-\mathrm{mL}$ tube (Falcon ${ }^{\mathrm{TM}} 15 \mathrm{~mL}$ Conical Centrifuge Tubes), which was then autoclaved at $121{ }^{\circ} \mathrm{C}$ for 15 min. For $S$. aureus, a liquid culture medium was prepared with peptone $[1.0 \mathrm{wt} \%$, Becton, Dickinson and Company (BD)], yeast extract ( $0.5 \mathrm{wt} \%, \mathrm{BD})$, and sodium 
chloride $(0.5 \mathrm{wt} \%)$. For E. coli, peptone $(1.0 \mathrm{wt} \%)$ from $\mathrm{BD}$, yeast extract $(0.5 \mathrm{wt} \%)$, and sodium chloride $(0.5$ wt $\%$ ) were used to prepare a liquid culture medium. For $S$. mutans, the liquid culture medium was prepared from Trypticase ${ }^{\mathrm{TM}}$ Soy Broth (Soybean-Casein Digest Medium) (3.0 wt \%, BD) and yeast extract $(0.3 \mathrm{wt} \%)$. These liquid culture media were used to adjust bacterial concentration to $1.0 \pm 0.3 \times 10^{5}$ colony-forming units $(\mathrm{CFU}) / \mathrm{mL}$ for $S$. aureus and E. coli, $1.67 \times 10^{8} \mathrm{CFU} / \mathrm{mL}$ for $S$. mutans, respectively. A sample was inoculated with $0.10 \mathrm{~mL}$ of a bacterial suspension and tightly sealed. Culturing conditions were $37{ }^{\circ} \mathrm{C}$ for $18 \mathrm{~h}$ for $S$. aureus and E. coli and $37{ }^{\circ} \mathrm{C}$ for $48 \mathrm{~h}$ for $S$. mutans.

Sodium chloride $(0.85 \mathrm{wt} \%)$ and Tween $80(0.20 \mathrm{wt} \%)$ from Santa Cruz Biotechnology, Inc., were added into and incubated in the culture tube (Falcon ${ }^{\mathrm{TM}} 15 \mathrm{~mL}$ Conical Centrifuge Tubes) for adjustment to a desired bacterial concentration, followed by the addition of $10 \mathrm{~mL}$ of physiological saline for washing and shaking to disperse the bacteria. Physiological saline prepared from sodium chloride $(0.85 \mathrm{wt} \%)$ was added to each stock bacterial suspension for up to $10^{7}$-fold dilution of a given concentration.

Mannitol salt agar (Code 05236, Nissui Pharmaceutical Co., Ltd., Tokyo, Japan) plates adjusted to $11.1 \mathrm{wt} \%$ were prepared for S. aureus; MacConkey agar (Code 05036, Nissui Pharmaceutical Co., Ltd., Tokyo, Japan) plates adjusted to $5.0 \mathrm{wt} \%$ were prepared for E. coli; and agar plates with Trypticase ${ }^{\mathrm{TM}}$ Soy Broth adjusted to $3.0 \mathrm{wt} \%$, yeast extract adjusted to $0.3 \mathrm{wt} \%$, and agar powder (Code 018-08721, Wako Pure Chemical Industries, Ltd., Osaka, Japan) for bacterial media adjusted to $1.5 \mathrm{wt} \%$ were prepared for $S$. mutans. A plate was inoculated with a bacterial suspension diluted to desired concentrations. Inoculation involved dropping $5 \mu \mathrm{L}$ of a diluted bacterial suspension at five spots in each of the four quadrants of a plate [5-7, 10].

The plates were inverted and incubated at $37{ }^{\circ} \mathrm{C}$ for $20 \mathrm{~h}$ for $S$. aureus and E. coli (personal Incubator PIC-100, AS ONE, Osaka, Japan) and at $37{ }^{\circ} \mathrm{C}$ for $44 \mathrm{~h}$ for $S$. mutans. Colonies were counted after the incubation, and each number was multiplied by a respective dilution factor to obtain a viable cell count. An Anello Pack Gas Detection System by Mitsubishi Gas Chemical, Inc., was employed for the incubation of $S$. mutans, which is an anaerobic bacterium.

\section{Evaluation of deodorant ability}

A quantity of $400 \mathrm{~mL}$ each of acetic acid, ammonia, trimethylamine, and acetaldehyde was placed in a separate 500-mL glass bottle (AS ONE, Osaka, Japan) at room temperature. A $2.0-\mathrm{mL}$ syringe (All glass Type, AS ONE, Osaka, Japan) was used to take an appropriate amount of saturating vapor from the bottle via suction. Each of the highly concentrated odorous gases mentioned above was injected via a syringe into a separate Tedlar bag (AS ONE, Osaka, Japan) containing $20 \mathrm{~L}$ of pure air. Additional pure air was added to the Tedlar bags to adjust the concentrations of the gases to the following values: acetic acid, $25 \pm 0.5 \mathrm{ppm}$; ammonia, $60 \pm 2 \mathrm{ppm}$; trimethylamine, $60 \pm 2 \mathrm{ppm}$; and acetaldehyde, $20 \pm 2 \mathrm{ppm}$.

An $1 \pm 0.0001 \mathrm{~g}$ sample of paper was placed in a $5-\mathrm{L}$ Tedlar bag, to which $3.0 \mathrm{~L}$ of an odorous gas was transferred at a flow rate of $500 \mathrm{~mL} / \mathrm{min}$ with a measuring pump (DP0105, Nitto Kohki Co., Ltd., Tokyo, Japan). The gas concentration in the bag was measured using a Gas Sampling Pump (GV-100 Type, Gastec Corporation, Kanagawa, Japan) at $10 \mathrm{~min}, 30 \mathrm{~min}, 1,3,6$, and $24 \mathrm{~h}$ from the time of the gas injection into the Tedlar bag. The initial concentration of the gas was converted to $100 \%$ and a plot of the odor residual rate was mapped against time. The odor residual rate of the sample was calculated by the Eq. (1) given below [7, 10, 11].

$$
\text { Odorresidual rate }(\%)=\frac{\text { Themeasuredgasconcentration }}{\text { Initialconcentration }} \times 100 \text {. }
$$

\section{Observation under a scanning electron microscope}

A carbon tape for vapor deposition was pasted on a stage for electron microscopy, and a sample was fixed onto the tape. After the fixed sample was dried at room temperature for $24 \mathrm{~h}$, gold was vapor-deposited on the sample using an ion sputterer (E-1010, Hitachi High-Technologies Corp., Tokyo, Japan). An S-3000 N scanning electron microscope (Hitachi Science Systems Co., Ltd., Tokyo, Japan) was then used to observe the surface of the crushed horse chestnut's shell and papers. The SEM was operated at an accelerating voltage of $20 \mathrm{kV}$.

\section{Mechanical properties of paper}

The tensile strength of a test piece was tested under dry conditions in compliance with JIS-P8113 [12, 13]. The test piece was cut to a width of $15.0 \pm 0.1 \mathrm{~mm}$ and length of $250 \pm 1 \mathrm{~mm}$. The maximum load at failure of the test piece was measured with a tensile tester at a chuck interval of $180 \pm 1 \mathrm{~mm}$ and tension speed of $20 \mathrm{~mm} / \mathrm{min}$. The elongation when the test sample broke owing to tension was measured, and the tensile elongation ratio was calculated. In both tests, the measurement was carried out in the longitudinal direction only. In addition, the test piece was swollen with water for $1 \mathrm{~h}$. The test piece was tested under wet conditions in the same manner as described above. 
The Clark stiffness of the paper was measured in compliance with JIS-P8143 [14]. A test piece with a width of $30 \mathrm{~mm}$ and length of $80 \mathrm{~mm}$ was sandwiched between two rollers that can be rotated in the clockwise and counterclockwise directions. The critical length of the test piece was measured; the critical length is the length of the test piece when it bends at an angle (critical turning angle) of $90^{\circ}$, caused by its empty weight, with the rotation of the rollers. The Clark stiffness is calculated by raising the critical length to the third power and dividing by 100 ; this value represents the bending resistance of a test piece owing to its empty weight - that is, the hardness of paper.

Using the following formula (2), the Clark stiffness was calculated:

Clark stiffness $S=\frac{L^{3}}{100}$.

Here, $S$ is the Clark stiffness; $L$ is the critical length $(\mathrm{cm})$.

\section{Gurley-type air resistance}

The air resistance of the paper was measured using a Gurley testing machine in compliance with JIS-P8117 $[13,15]$. First, $300 \mathrm{~mL}$ of air that was compressed by the weight of an inner cylinder was passed through a $50 \mathrm{~mm} \times 50 \mathrm{~mm}$ test piece fixed to a tightening plate with a circular hole having a diameter of $28.6 \pm 0.1 \mathrm{~mm}$ (air permeation area: $642 \mathrm{~mm}^{2}$ ). The time period required for $300 \mathrm{ml}$ of the compressed air to pass through the hole was represented by $t(\mathrm{~s})$. A shorter $t$ meant higher air permeability for the paper.

\section{Results and discussion}

\section{Shapes of particles of ground horse chestnut seed}

Electron microscopic analysis at $700 \times$ magnification was carried out for the ground outer theca as well as ground sarcocarp and endodermis of horse chestnut seed, which were described in section "Samples" (Fig. 2). Many pieces of the ground sarcocarp of horse chestnut seed were found to have a round shape $10-20 \mu \mathrm{m}$ in diameter (Fig. 2a). Furthermore, there were long ground pieces of the endodermis indicated by a circle of a dotted line (Fig. 2a).

On the other hand, pieces of the ground outer theca were found to be relatively large: approximately $30 \mu \mathrm{m}$ in width and approximately $100 \mu \mathrm{m}$ in length (Fig. 2b). Unlike the ground sarcocarp, ground pieces of the outer theca were not round. As described above, the outer theca was so hard to grind that its ground pieces remained relatively large,
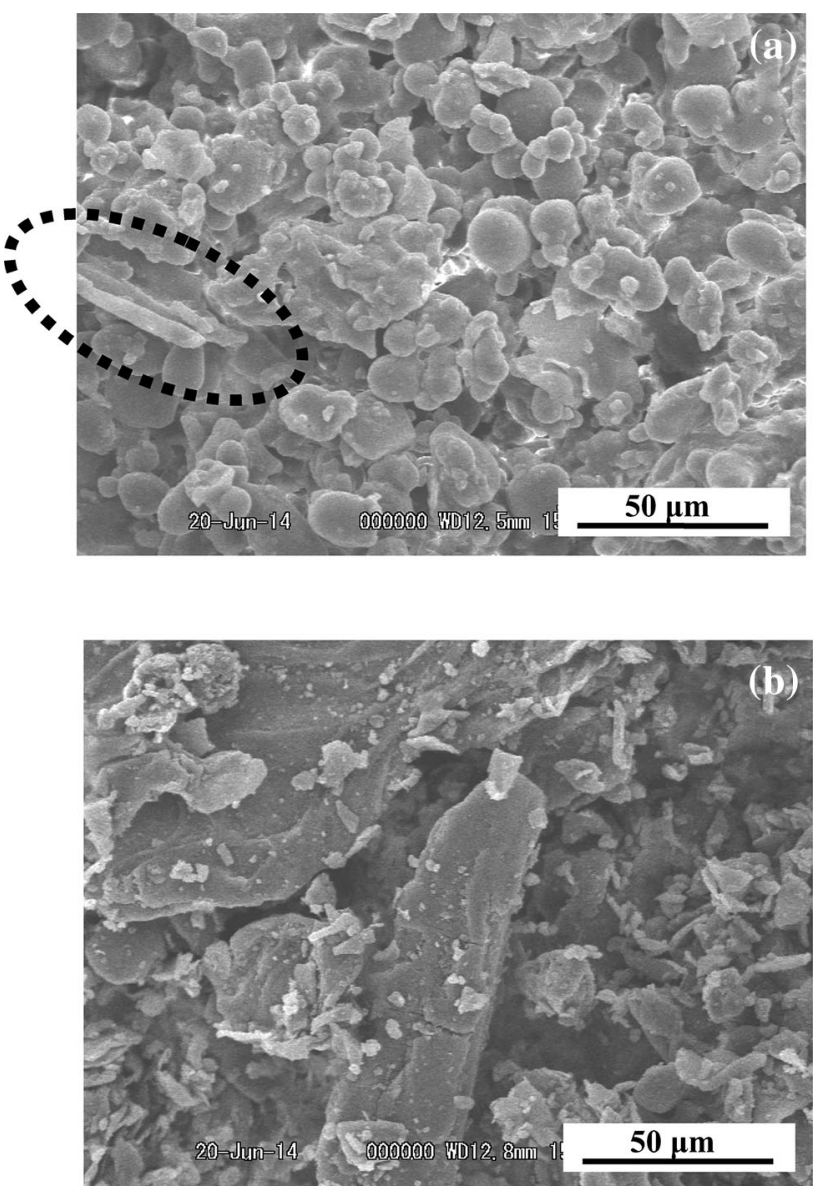

Fig. 2 Scanning electron micrographs of ground horse chestnut seed $(\times 700)$. Acceleration Voltage: $20 \mathrm{kV}$. a sarcocarp and endodermis, b outer theca. The circle of a broken curve shows parts of the endodermis

whereas the sarcocarp of horse chestnut seed was ground to relatively small pieces.

\section{Antibacterial activities of ground horse chestnut seed}

Gram-positive S. aureus, Gram-negative E. coli, and anaerobic $S$. mutans were used to conduct assays of antibacterial activities of the ground seed. Table 1 shows the results. The results revealed that all three bacteria species yielded growth values of 0.5 or higher, which is the JIS standard for the assay [9]. In other words, all three species grew without any problem in the antibacterial assays.

$S$. aureus grew from a viable count of $1.00 \times 10^{5} \mathrm{CFU} /$ $\mathrm{mL}$ before incubation to that of $9.44 \times 10^{8} \mathrm{CFU} / \mathrm{mL}$ after $18 \mathrm{~h}$ of incubation in the culture medium alone. In short, this bacterium was found to proliferate roughly $10^{4}$-fold. In contrast, when inoculated on to the endodermis and seed of the horse chestnut seed, incubation decreased the viable 
Table 1 Antibacterial properties of the ground horse chestnut seed against different species of bacterium

\begin{tabular}{|c|c|c|c|c|c|}
\hline \multirow[t]{2}{*}{ Species of bacterium } & \multirow[t]{2}{*}{ Sample } & \multirow{2}{*}{$\begin{array}{l}\text { Incubation } \\
\text { time (h) }\end{array}$} & \multicolumn{2}{|c|}{ Antibacterial properties } & \multirow{2}{*}{$\begin{array}{l}\text { Bacteria yielded } \\
\text { growth values }\end{array}$} \\
\hline & & & $\begin{array}{l}\text { Viable bacteria } \\
\left(\mathrm{CFU}^{\mathrm{a}} / \mathrm{mL}\right)\end{array}$ & $\begin{array}{l}\text { Antibacterial } \\
\text { activity value }^{c}\end{array}$ & \\
\hline \multirow[t]{4}{*}{ Staphylococcus aureus } & Before incubation & 0 & $1.00 \times 10^{5}$ & - & \\
\hline & Sarcocarp and endodermis & 18 & $3.20 \times 10^{4}$ & 4.47 & \\
\hline & Outer theca & 18 & $\mathrm{ND}^{\mathrm{b}}$ & $\infty$ & \\
\hline & Only bacteria & 18 & $9.44 \times 10^{8}$ & - & 3.98 \\
\hline \multirow[t]{4}{*}{ Escherichia coli } & Before incubation & 0 & $1.00 \times 10^{5}$ & - & \\
\hline & Sarcocarp and endodermis & 18 & ND & $\infty$ & \\
\hline & Outer theca & 18 & ND & $\infty$ & \\
\hline & Only bacteria & 18 & $6.88 \times 10^{8}$ & - & 3.84 \\
\hline \multirow[t]{4}{*}{ Streptococcus mutans } & Before incubation & 0 & $1.67 \times 10^{8}$ & - & \\
\hline & Sarcocarp and endodermis & 48 & $1.20 \times 10^{7}$ & 2.73 & \\
\hline & Outer theca & 48 & $6.73 \times 10^{4}$ & 4.97 & \\
\hline & Only bacteria & 48 & $6.31 \times 10^{10}$ & - & 1.58 \\
\hline
\end{tabular}

${ }^{a}$ Colony-forming units

b Not Detected

${ }^{\mathrm{c}}$ Antibacterial activity value $=\left(\log C_{\mathrm{t}}-\log C_{0}\right)-\left(\log T_{\mathrm{t}}-\log T_{0}\right)$; where $\log C_{t} \log ($ average (viable cell count after incubation of bacteria alone for a given period)); $\log C_{0} \log \left(\right.$ average (viable cell count before incubation)); $\log T_{t} \log ($ average (viable cell count after incubation of the inoculated samples for a given period)); $\log T_{0} \log ($ average (viable cell count of the samples immediately after inoculation))

${ }^{\mathrm{d}}$ Bacteria yielded growth values $=\log C_{\mathrm{t}}-\log C_{0}$

count to $3.20 \times 10^{4} \mathrm{CFU} / \mathrm{mL}$, or approximately $1 / 10$ of the viable count before the incubation. Considering the viable count of $9.44 \times 10^{8} \mathrm{CFU} / \mathrm{mL}$ when the bacterium alone was incubated, the effect was found to correspond to approximately 1000 -fold decrease in the cell count. The outer theca of the horse chestnut seed did not show any viable cells of the bacterium at all. In short, the bacterium was effectively killed. These data indicated that horse chestnut seed, especially the outer theca, has antibacterial properties.

Neither the sarcocarp nor endodermis of horse chestnut seed showed any viable cells of E. coli, a Gram-negative bacterium, after $18 \mathrm{~h}$ of incubation. These data showed that every part of the horse chestnut seed has a strong antibacterial activity toward E. coli.

The viable cell count of $S$. mutans, a Gram-positive anaerobic bacterium, was $1.67 \times 10^{8} \mathrm{CFU} / \mathrm{mL}$ before incubation and $6.31 \times 10^{10} \mathrm{CFU} / \mathrm{mL}$ after $48 \mathrm{~h}$ of incubation in the culture medium alone. In brief, when the bacterium alone was incubated, the viable count increased 100 -fold. In contrast, when the bacterium was inoculated onto the endodermis and sarcocarp of the horse chestnut seed, the viable cell count decreased to $1.2 \times 10^{7} \mathrm{CFU} / \mathrm{mL}$ or 1/10-fold. Furthermore, after inoculation onto the outer theca of horse chestnut seed, the viable cell count decreased to $6.73 \times 10^{4} \mathrm{CFU} / \mathrm{mL}$. Compared with the incubation of the bacterium alone, the viable count was found to decrease by as much as $1 / 10,000$-fold. In conclusion, the outer theca of the horse chestnut seed clearly possesses excellent antibacterial activity toward $S$. mutans.

Such a difference in antibacterial activity between assays of $S$. aureus and of E. coli was assumed to result from the difference in bacterial cell wall. That is, S. aureus and S. mutans, both of which are Gram-positive, have a thick layer of the cell wall mostly consisting of peptidoglycan, whereas Gram-negative E. coli has a cell wall containing a small amount of peptidoglycan. In general, Gram-positive bacteria have a peptidoglycan layer as thick as $20-80 \mathrm{~nm}$, whereas Gram-negative bacteria have a layer $7-8 \mathrm{~nm}$ thick, i.e., Gram-positive bacteria have a far thicker cell wall. The dry weight of the bacterial cell wall is only $10 \%$ in Gram-negative bacteria. In contrast, it is as high as $90 \%$ in Gram-positive bacteria. As a consequence, the antibacterial activity of horse chestnut seed more easily damaged the cell wall of Gram-negative bacteria and more easily acted on $E$. coli in particular.

JIS stipulates that a value of 2.0 or higher in an assay of antibacterial activity (a kill ratio of $99 \%$ or higher) means an antibacterial effect [9]. Horse chestnut seed yielded high values of antibacterial activity, higher than 2.0, toward all 
Table 2 Soluble polyphenol concentration

\begin{tabular}{lll}
\hline & mg catechin eq./100 gDW & Standard error $(n=6)$ \\
\hline Sarcocarp and endodermis & 1184 & 25 \\
Outer theca & 3476 & 55 \\
Paper containing 20wt\% of theca & 36.0 & 0.5 \\
Paper containing 40wt $\%$ of theca & 96.8 & 2.8 \\
Paper containing 60wt\% of theca & 172.3 & 14.2 \\
\hline
\end{tabular}

the bacteria tested. The horse chestnut seed was found to have high antibacterial effects also on the basis of the JIS standard.

The difference in antibacterial activity between the "sarcocarp and endodermis" of horse chestnut and its "outer theca" is believed to be due to their different components. Generally, polyphenols are thought to be closely involved in antibacterial activity. Thus, we examined the soluble polyphenol concentration in the "sarcocarp and endodermis" and "outer theca", and the results are presented in Table 2. The results showed that the soluble polyphenol concentration was $3476 \mathrm{mg}$ catechin eq./ $100 \mathrm{gDW}$ in the "outer theca" and $1184 \mathrm{mg}$ catechin eq./ $100 \mathrm{gDW}$ in the "sarcocarp and endodermis". Thus, the polyphenol content of the "outer theca" was approximately three times higher than that of the "sarcocarp and endodermis". Table 2 also shows the soluble polyphenol concentration in compounded paper, which is discussed later.

To study the mechanism behind the antibacterial activity of horse chestnut seed, it was decided to measure ORAC values as an indicator of antioxidant activity. The concept of ORAC was developed by the researchers at the USDA and National Institute on Aging. ORAC is a numerical value showing oxygen radical absorbance capacity (antioxidant capacity). Two measurements must be made: one with a substance in a ground state and the other with an extract (Fig. 3). The results revealed that both the ground outer theca of the horse chestnut seed and its extract yielded approximately 4.2-fold greater ORAC values than the sarcocarp and endodermis did. The higher antibacterial activity in the "outer theca" than in the

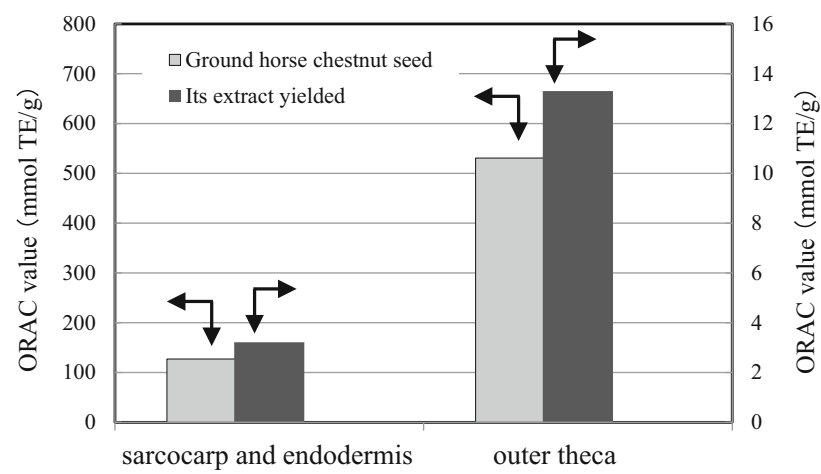

Fig. 3 ORAC values per gram for ground parts of the horse chestnut seed and extracts "sarcocarp and endodermis" was believed to be due to the difference in the soluble polyphenol concentration. The polyphenols contained in them potentially acted as antioxidants [16, 17]. In brief, antioxidant activity damaged the peptidoglycan layer [18] of the bacterial cell wall and thus suppressed bacterial growth. Nevertheless, it cannot necessarily be stated that antioxidant function alone is responsible for the antibacterial activity. Some receptors on the surface of bacterial cells may conceivably be also involved [19]. Moreover, ORAC was approximately 40-fold higher in the ground state than in the extract. This finding revealed that the extraction removed approximately $2.5 \%$ of antibacterial activity from the ground sample.

\section{Deodorizing activity of the outer theca of horse chestnut seed}

It was decided to focus on the outer theca, which showed high antioxidant and antibacterial activities. We also wanted to examine the deodorizing activity of the outer theca against various kinds of odor. Odorous gases used in the deodorization assays were ammonia and trimethylamine as basic odors, acetic acid as an acidic odor, and acetaldehyde as a neutral odor.

Figure 4 shows how the odor residual rate depended on time. The results revealed that the outer theca of horse chestnut seed effectively deodorized such a basic odor as ammonia and trimethylamine as well as acetic acid, which is an acidic odor. The odor residual rate of ammonia gas in particular decreased down to $0 \%$ in only $10 \mathrm{~min}$. This amazing effect should be marked for further analysis.

Concerning the mechanism of deodorization of ammonia gas by the ground outer theca of horse chestnut seed, it was assumed that hydroxyl groups of polyphenol in the outer theca formed hydrogen bonds with ammonia molecules to form a salt, which was then released into moisture in the air. To sum up, we assumed that some $\mathrm{OH}$ groups of polyphenol in the ground outer theca were chemically transformed to $\mathrm{O}^{-} \mathrm{NH}_{4}^{+}$to incorporate ammonia gas into its molecular structure [7].

In addition, the odor residual rate for acetic acid gas was found to substantially decrease to $1.9 \%$ in only $30 \mathrm{~min}$. In short, the ground outer theca was also found to possess good deodorization capacity against acidic odor. 


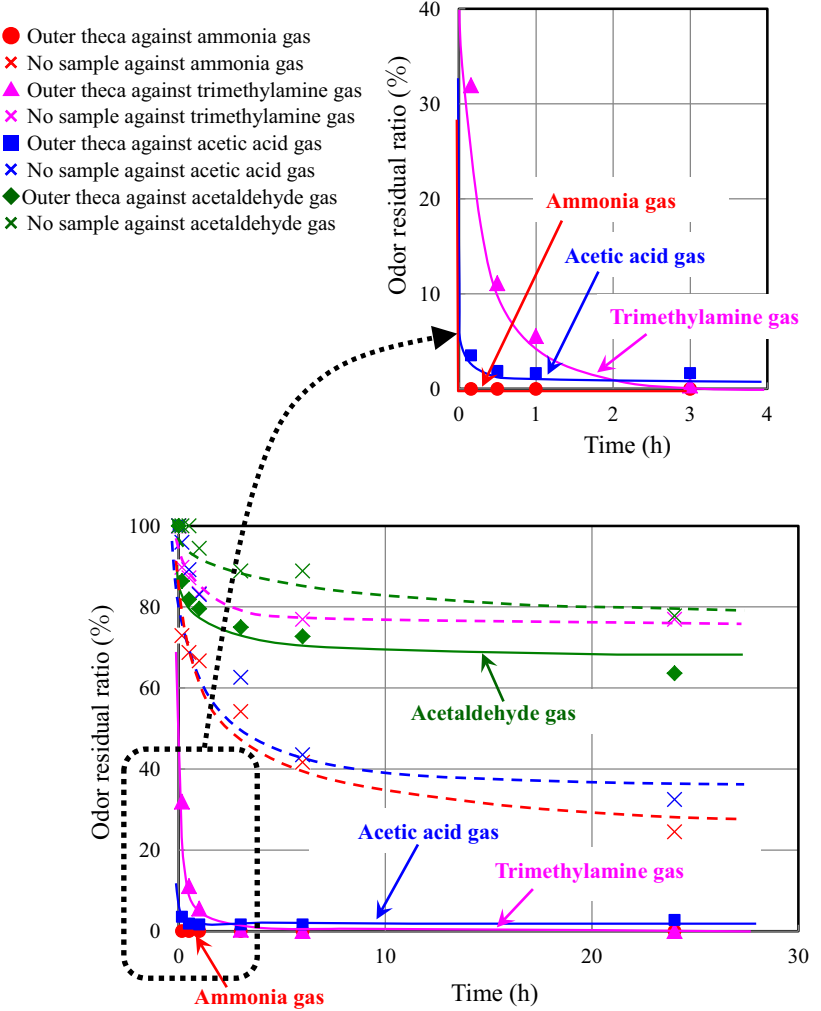

Fig. 4 Time course analyses of various odorous gases showing odor residual ratios for the ground outer theca of the horse chestnut seed

According to the Weber-Fechner law, sensory intensity of an odor is proportional to the logarithm of the concentration of the odorant. That is, a 10-fold increase in odorant concentration is perceived as approximately two-fold increase in the intensity of the odor. Conversely, one tenth of the concentration is perceived half as intense in terms of odor. In conclusion, ground horse chestnut seed was found to halve the sensory intensity of a basic odor and an acidic odor. In stark contrast, the ground theca of horse chestnut was found to have a negligible deodorizing activity toward acetaldehyde, a neutral odor.

\section{Performance of compounded paper containing the theca of horse chestnut seed}

As described above, good antibacterial and deodorizing activities were found in the ground outer theca of horse chestnut seed. A product with a large specific surface area may be considered suitable for effective practical use of these properties. An attempt was made to prepare compounded paper as a way to effectively utilize the outer theca of horse chestnut seed. The ground outer theca was blended with pulp in ratios of 20,40 , or $60 \mathrm{wt} \%$ to prepare compounded paper by the traditional Washi paper-making method. Stable production of compounded paper with uniform weight distribution was achieved.

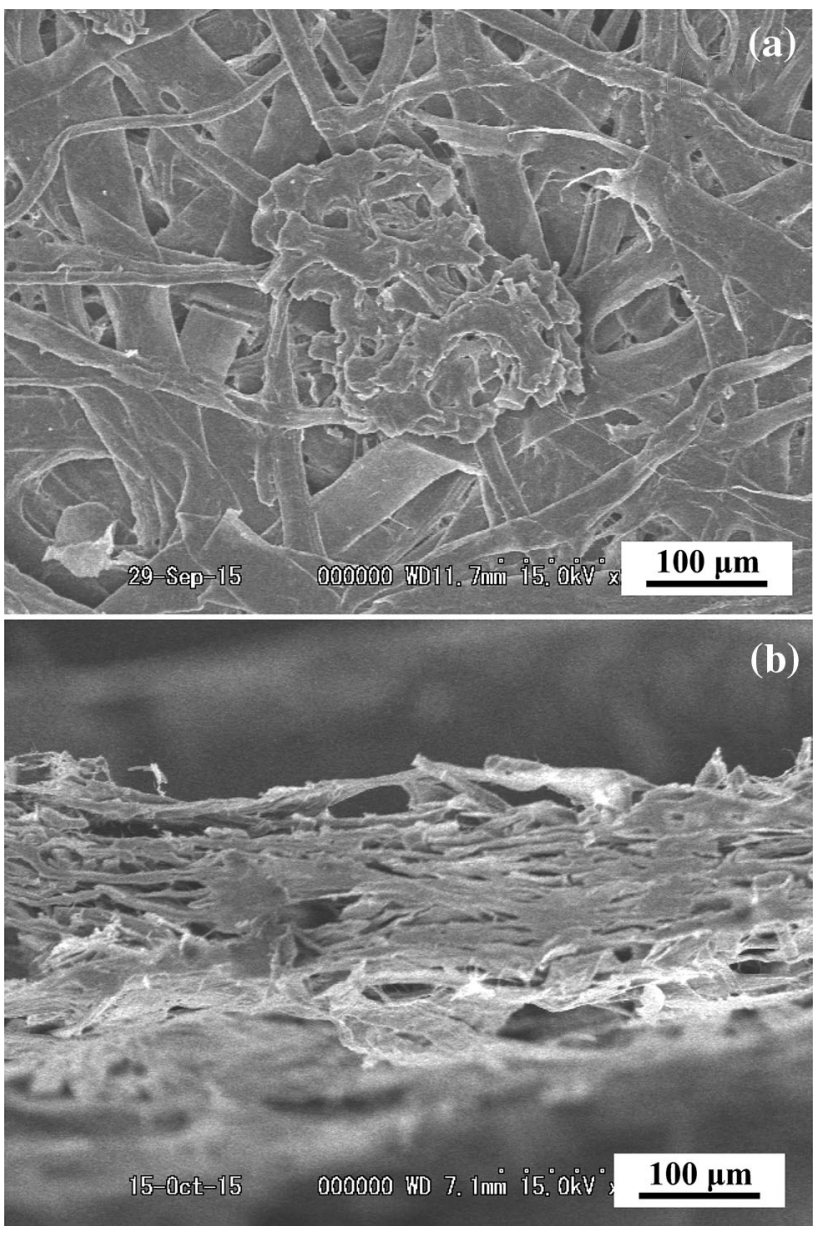

Fig. 5 Scanning electron micrographs of compounded papers containing the ground theca at a $60 \mathrm{wt} \%$ proportion $(\times 200)$. Acceleration voltage: $20 \mathrm{kV}$. a Surface, b cross-section

Electron microscopy was used to examine the structure of compounded paper containing the ground outer theca of horse chestnut seed. Figure 5a, b show the surface and cross-section of compounded paper containing the ground theca at a $60 \mathrm{wt} \%$ proportion. The ground outer theca was found to be present as lumps in some parts of the pulp fiber network. That is, the ground outer theca was not found to be completely uniformly distributed among pulp fibers. Nonetheless, the ground theca did not come off when the compounded paper was touched with a hand.

Subsequently, the compounded paper prepared as described above was examined for antibacterial activity. E. coli, toward which the ground theca showed the highest antibacterial activity, was used in the assays of antibacterial activity. Each sample was inoculated with a bacterial suspension at a concentration adjusted to $10^{5} \mathrm{CFU} / \mathrm{mL}$ and incubated for $20 \mathrm{~h}$ before viable cell counts were determined.

Figure 6 presents photographs of the culture medium incubated for $20 \mathrm{~h}$. Many colonies were observed in cases 
Fig. 6 Antibacterial properties of compounded papers containing the ground outer theca for different proportions. Photographs of the culture medium incubated for $20 \mathrm{~h}$ for Escherichia coli

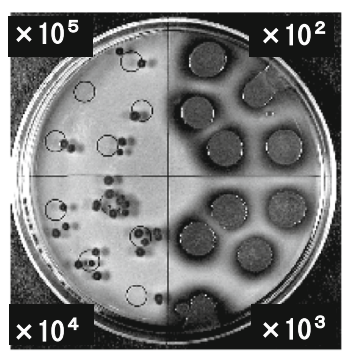

0wt\% (Only pulp)

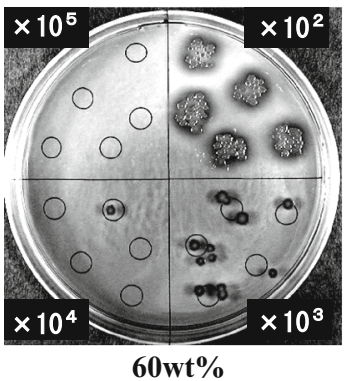

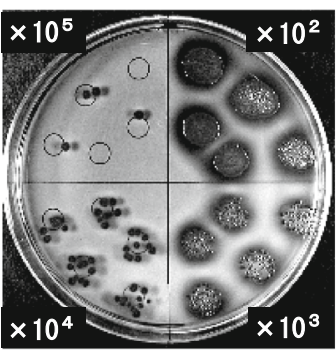

$20 w t \%$

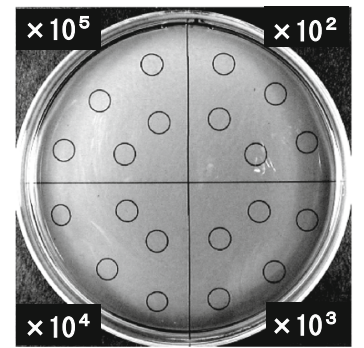

$100 \mathrm{wt} \%$ (Only ground theca)
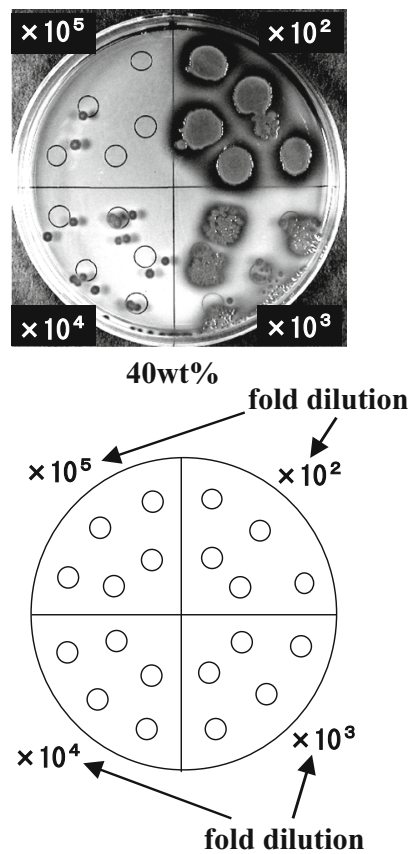

of compounded paper containing $100 \mathrm{wt} \%$ pulp or only 20 wt $\%$ of the ground theca. Especially in the quadrants of $10^{2}$ - or $10^{3}$-fold dilutions colonies were so numerous that they were found to overlap and form aggregates. In the case of compounded paper containing $40 \mathrm{wt} \%$ of ground theca, the number of colonies was somewhat smaller than that in cases of 0 or $20 \mathrm{wt} \%$ of the ground theca. The number of colonies was found to further decrease in the case of compounded paper containing $60 \mathrm{wt} \%$ of the ground theca. In particular, a comparison of $10^{3}$-fold dilution quadrants clearly showed that the number of colonies in the case of $60 \mathrm{wt} \%$ of the ground theca was smaller than in cases of other compounded paper samples containing a smaller proportion of the ground theca. In contrast, no colonies were detected in the culture medium containing the ground theca alone. That is, the ground theca itself was found to possess very high antibacterial activity.

Viable cell counts obtained in the antibacterial activity assays were analyzed next (Table 3 ). The data revealed that the viable count was $1.10 \times 10^{7} \mathrm{CFU} / \mathrm{mL}$ in the case of compounded paper containing $20 \mathrm{wt} \%$ of the ground theca in contrast to $1.58 \times 10^{7} \mathrm{CFU} / \mathrm{mL}$ in the case of compounded paper of $100 \mathrm{wt} \%$ pulp, revealing little or no difference. When the proportion of the ground theca increased to $40 \mathrm{wt} \%$, viable counts decreased a little, down to $9.20 \times 10^{6} \mathrm{CFU} / \mathrm{mL}$. When the proportion of the ground theca further increased to $60 \mathrm{wt} \%$, the viable cell count was found to decrease to $3.96 \times 10^{5} \mathrm{CFU} / \mathrm{mL}$. To sum up, the antibacterial activity increased with the increasing proportion of ground theca in compounded paper to suppress bacterial growth 1/100-fold. Nevertheless, a comparison with the case of the ground theca alone indicated that the viable cell count was very high in case of compounded paper.

The traditional Washi paper-making method adopted for the preparation of compounded paper was assumed to be responsible for the observed lack of the effect. In other words, effective antibacterial components may be eliminated or destroyed in that paper-making process. Therefore, the soluble polyphenol concentrations of those compounded papers were examined (Table 2). The results showed that compounded papers with higher antibacterial activities had a higher soluble polyphenol concentration. However, the compounded papers were found to have much lower soluble polyphenol concentration than the ground seed of horse chestnut. To use compounded paper containing horse chestnut seed in applications that require high antibacterial activity, it may be helpful to do further adjustments and optimizations, such as adopting a manufacturing process other than the traditional Washi papermaking method.

After that, compounded paper containing the ground outer theca of horse chestnut seed was examined for deodorizing activity. In the testing, we used ammonia gas, toward which the ground outer theca had shown high deodorizing activity. Figure 7 presents the results. Compounded paper containing $60 \mathrm{wt} \%$ of the ground outer theca of horse chestnut seed showed a strong deodorizing activity: the odor residual rate was only $0.8 \%$ within only $30 \mathrm{~min}$. Furthermore, the odor residual rate was found to be low, at $9.2 \%$, after $30 \mathrm{~min}$ even in the case of 
Table 3 Antibacterial properties of compounded papers containing the ground theca against Escherichia coli

\begin{tabular}{|c|c|c|c|c|}
\hline \multirow[t]{2}{*}{ Sample } & \multirow{2}{*}{$\begin{array}{l}\text { Incubation } \\
\text { time }(\mathrm{h})\end{array}$} & \multicolumn{2}{|c|}{ Antibacterial properties } & \multirow{2}{*}{$\begin{array}{l}\text { Bacteria yielded } \\
\text { growth values }\end{array}$} \\
\hline & & $\begin{array}{l}\text { Viable bacteria } \\
\left(\mathrm{CFU}^{\mathrm{a}} / \mathrm{ml}\right)\end{array}$ & $\begin{array}{l}\text { Antibacterial } \\
\text { activity value }^{c}\end{array}$ & \\
\hline Before incubation & 0 & $1.00 \times 10^{5}$ & - & \\
\hline 100 wt $\%$ pulp paper & 18 & $1.58 \times 10^{7}$ & -0.05 & \\
\hline Paper containing $20 \mathrm{wt} \%$ of theca & 18 & $1.10 \times 10^{7}$ & 0.11 & \\
\hline Paper containing $40 \mathrm{wt} \%$ of theca & 18 & $9.20 \times 10^{6}$ & 0.19 & \\
\hline Paper containing $60 \mathrm{wt} \%$ of theca & 18 & $3.96 \times 10^{5}$ & 1.55 & \\
\hline Only ground outer theca & 18 & $\mathrm{ND}^{\mathrm{b}}$ & $\infty$ & \\
\hline Only bacteria & 18 & $1.42 \times 10^{7}$ & - & 2.15 \\
\hline
\end{tabular}

a Colony-forming units

b Not detected

c Antibacterial activity value $=\left(\log C_{\mathrm{t}}-\log C_{0}\right)-\left(\log T_{\mathrm{t}}-\log T_{0}\right)$; where $\log C_{t} \log ($ average (viable cell count after incubation with $100 \mathrm{wt} \%$ pulp paper for a given period)); $\log C_{0} \log \left(\right.$ average (viable cell count before incubation)); $\log T_{t} \log ($ average (viable cell count after incubation of the inoculated samples for a given period)); $\log T_{0} \log ($ average (viable cell count of the samples immediately after inoculation))

${ }^{\mathrm{d}}$ Bacteria yielded growth values $=\log C_{\mathrm{t}}-\log C_{0}$

compounded paper with ground-theca content of $20 \mathrm{wt} \%$. Ammonia gas is said to be faintly odorous and detectable even at $1 \mathrm{ppm}$ (detectable threshold concentration). As for the method for evaluation of deodorizing textiles, Japan Textile Evaluation Technology Council states that a deodorizing textile needs to decrease odor

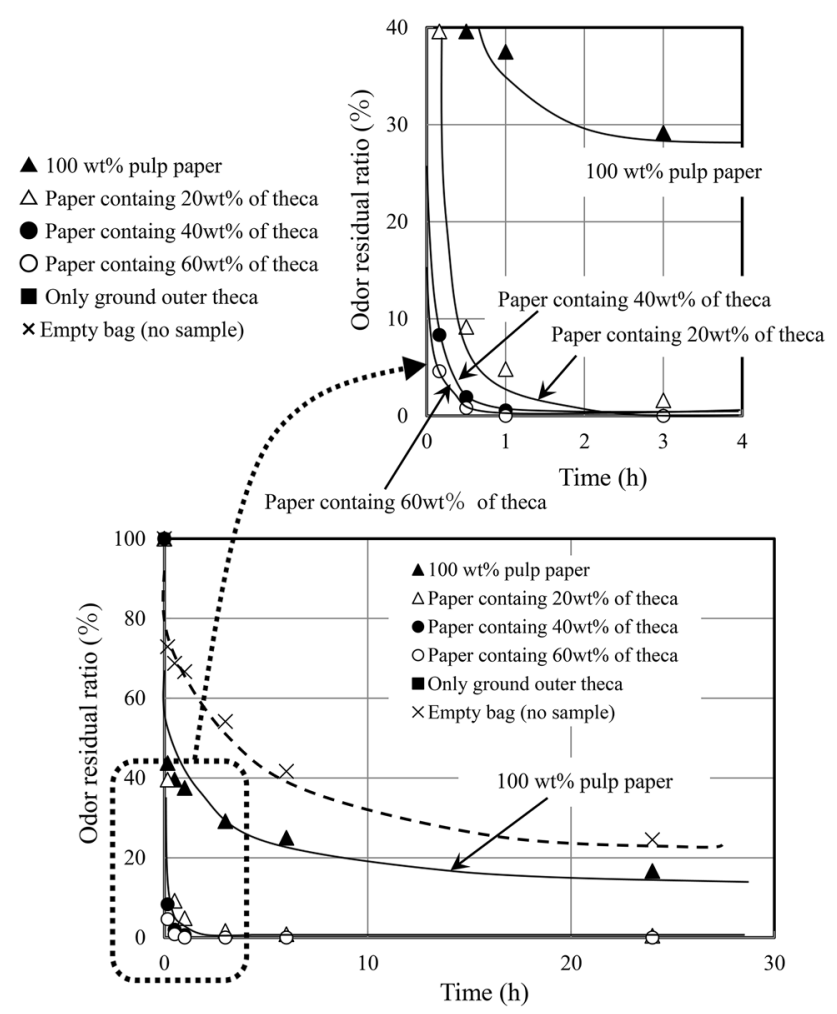

Fig. 7 Time course of ammonia gas showing an odor residual ratio for compounded paper containing the ground outer theca of the horse chestnut seed concentration of $>40 \mathrm{ppm}$ (which is said to be intensely odorous) to under a detectable threshold concentration within $24 \mathrm{~h}$ [20]. In addition, by this criterion, compounded paper containing the outer theca of horse chestnut seed can be said to possess an excellent deodorizing activity against ammonia gas.

\section{Mechanical properties of compounded paper containing the outer theca of horse chestnut seed}

Various applications may be considered for compounded paper with such excellent antibacterial and deodorizing activities. Nonetheless, practical applications of compounded paper often require certain mechanical properties and gas permeability. First, compounded paper samples with different proportions of the ground theca were examined for such mechanical properties as tensile strength, tensile elongation, and Clark stiffness. Figure 8 shows the results. All the properties were found to deteriorate with the increasing proportion of outer theca of horse chestnut seed. Mechanical properties for compounded paper were also found to worsen with the decreasing proportion of pulp fiber. That is, the ground outer theca was found to worsen mechanical properties.

Hence, to examine the fracture mechanism of compounded paper, a double logarithmic graph was prepared to identify a relation between fracture strength and Clark stiffness (Fig. 9). The results showed a single straight line without dependence on ground outer theca content. The slope was approximately $1 / 2$. According to Griffith's theory, Eq. (2) shown below describes fracture stress $\alpha_{\mathrm{b}}$ and elastic modulus $E$ in brittle fracture [20]. Crack length is represented by $c$, and surface 


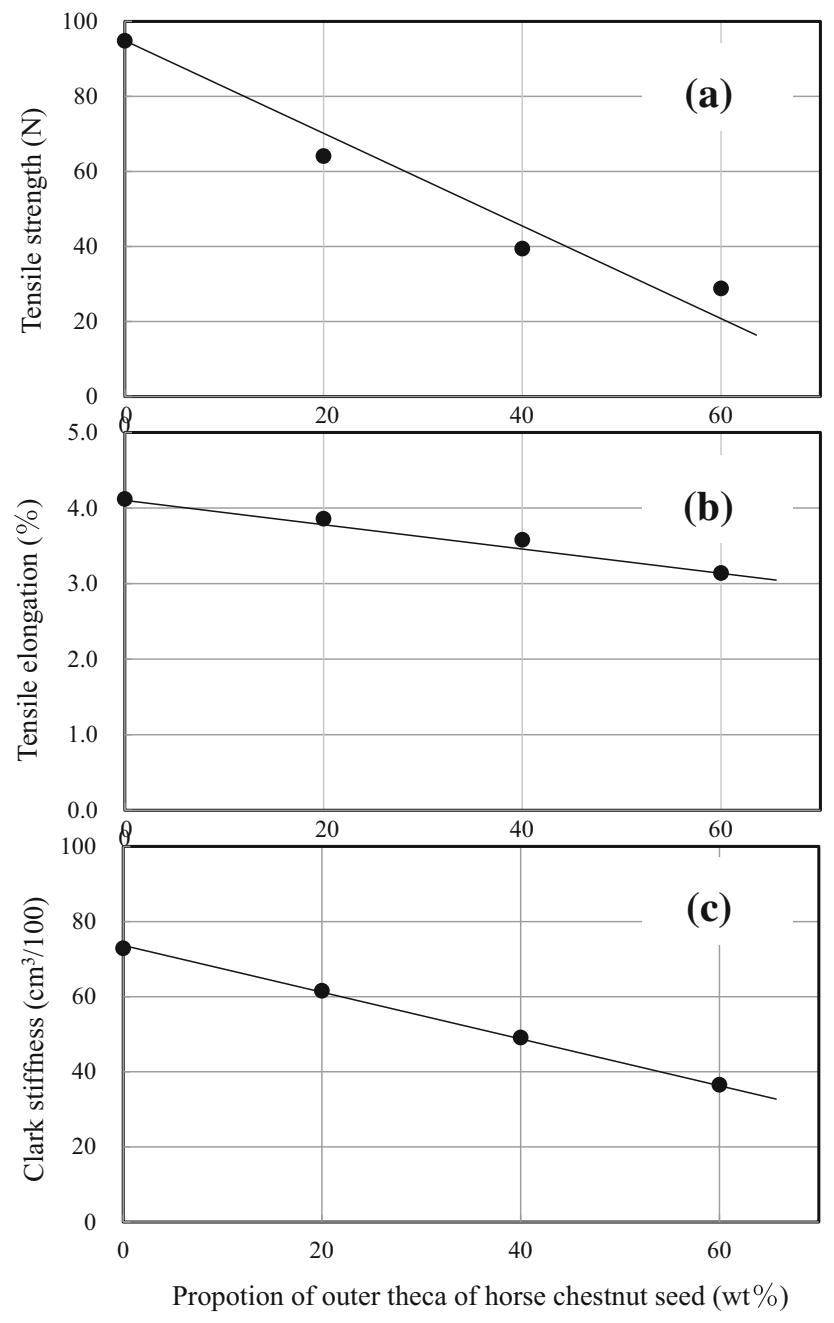

Fig. 8 Mechanical properties of compounded paper containing the outer theca of horse chestnut seed (temperature: $23{ }^{\circ} \mathrm{C}$, relative humidity: $50 \%$ ). a tensile strength, $\mathbf{b}$ tensile elongation and $\mathbf{c}$ Clark stiffness

energy per unit area for a new plane is denoted by $\gamma$ in the Eq. (3).

$\alpha_{b}=\sqrt{2 \gamma E / c}$.

In this experiment, tensile strength of compounded paper corresponded to stress at fracture, and Clark stiffness of compound paper corresponded to elastic modulus, which is resistance to transformation. The results depicted in Fig. 9 indicated that breaking strength of compounded paper containing the ground outer theca can be expressed by Griffith's fracture mechanism [21]. That is, Fig. 9 shows that fracture of compounded paper containing the ground outer theca originated in a crack caused by the ground theca inside compounded paper and extended along the development from the original crack. This finding is in agreement with the nonuniform distribution of the ground

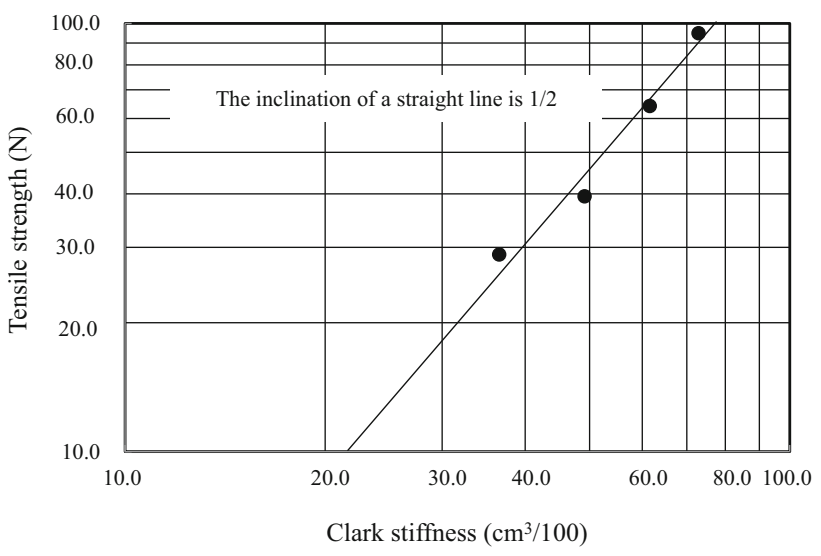

Fig. 9 Relationship between Clark stiffness and tensile strength of compounded paper containing the outer theca of horse chestnut seed

theca among pulp fibers, as determined by electron microscopy of compounded paper shown in Fig. 5.

Although mechanical properties of compounded paper deteriorated when the ground theca of horse chestnut seed was incorporated, they were also found to be maintained with pulp to some extent. Even at a $60 \mathrm{wt} \%$ proportion of the ground theca, tensile strength was $28.9 \mathrm{~N}$ and tensile elongation was $3.1 \%$ (Fig. 8). These values were found to be adequate for practical use. Furthermore, compounded paper with $20 \mathrm{wt} \%$ ground theca content had better mechanical properties, and adequate antibacterial and deodorizing activities. This finding revealed that compounded paper may be used in a wide range of applications.

Gas permeability was examined next. Figure 10 presents Gurley-type air resistance versus ground theca content. Air resistance, which corresponds to gas permeability of compounded paper, was found to decrease as the proportion of the ground theca of horse chestnut seed increased. In other

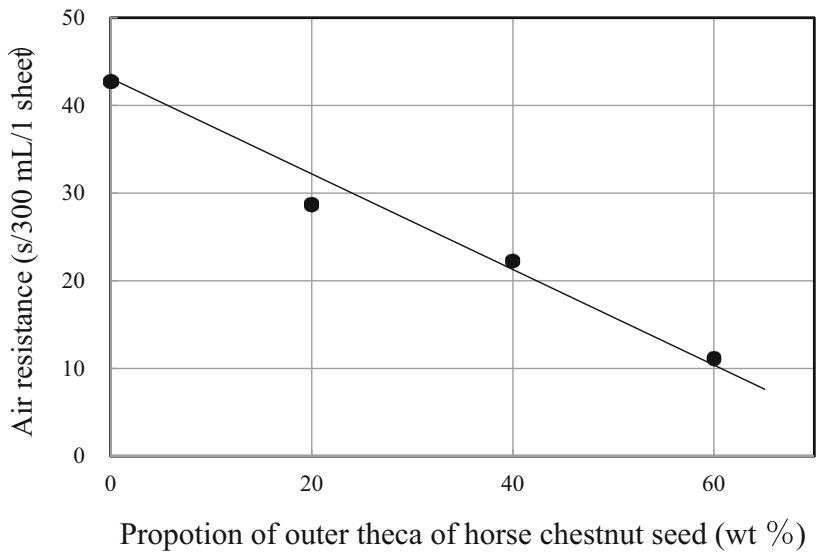

Fig. 10 Gurley-type air resistance of compounded paper containing the outer theca of horse chestnut seed (temperature: $23{ }^{\circ} \mathrm{C}$, relative humidity: $50 \%$ ) 
words, the greater the ground theca content is, the higher the gas permeability of compounded paper is. These data suggest that ground outer theca created small gaps among pulp fibers in compounded paper to increase gas permeability. This finding offers some directions for the development of practical applications of compounded paper containing the ground theca of horse chestnut seed. For example, high gas permeability may be useful in seed wrapping paper with excellent antibacterial activity. Furthermore, various applications may be feasible for multifunctional paper that has such properties as deodorizing and antibacterial activities.

\section{Conclusions}

For recycling of horse chestnut seed, it was separated into "sarcocarp and endodermis" and "outer theca"; each part was finely ground for analysis of its functions. Compounded paper containing outer theca was prepared and its properties were examined.

1. Ground horse chestnut seed showed excellent antibacterial activity toward $S$. aureus, E. coli, and S. mutans. Antibacterial activity is particularly strong against E. coli, which is a Gram-negative bacterium. The "outer theca" part showed a higher antibacterial activity than did the "sarcocarp and endodermis" part.

2. The ground theca of horse chestnut seed was found to have ORAC of $531 \mathrm{mmol} \mathrm{TE} / \mathrm{g}$ and to possess high antioxidant capacity. The reason why ground horse chestnut seed showed a high antibacterial activity may be its antioxidant properties. In other words, antioxidant activity was assumed to damage the peptidoglycan layer of the bacterial cell wall.

3. The outer theca of horse chestnut seed was found to have a high deodorizing activity against basic or acidic odors. For example, it was found to decrease ammonia gas to $0 \%$ (odor residual rate) only in $10 \mathrm{~min}$.

4. Compounded paper was prepared that contained the ground outer theca of horse chestnut seed blended with pulp fibers. The compounded paper was found to possess high deodorizing activity. Furthermore, although mechanical properties deteriorated as the proportion of the ground outer theca increased, these parameters remained adequate for practical applications.

\section{References}

1. Brockman CF, Merrilees R (1986) Trees of North America. Golden Press, New York
2. Mabberley DJ (1997) The plant book, 2nd edn. Cambridge University Press, Cambridge

3. Vander Wall SB (1990) Food hoarding in animals. University of Chicago Press, Chicago

4. Harborne JB, Baxter H (1993) Phytochemical dictionary. Taylor and Francis, London

5. Takahashi T, Aso Y, Kasai W, Kondo T (2010) Effect of light irradiation on the antibacterial activity of compounded papers containing wasted tea leaves. J Wood Sci 56(4):299-306

6. Takahashi T, Aso Y, Kasai W, Kondo T (2010) Improving the antibacterial activity in Staphylococcus aureus of composite sheets containing wasted tea leaves by roasting. J Wood Sci 56(5):403-410

7. Takahashi T, Aso Y, Kasai W, Kondo T (2011) Synergetic deodorant effect and antibacterial activity of composite paper containing waste tea leaves. J Wood Sci 57(4):308-316

8. Swain T, Hillis WE (1959) The phenolic constituents of Prunus domestica. I. The quantitative analysis of phenolic constituents. J Sci Food Agric 10:63-68

9. JIS-L1902 (2015) Determination of antibacterial activity and efficacy of textile products (in Japanese). Japanese Standard Association, Tokyo

10. Takahashi T, Aso Y, Yoshino K (2013) Incorporation of photocatalytic function into nonwoven polyester fabrics via impregnation with peroxo titanic acid solution. J Mater Sci 48(23):8199-8208

11. Takahashi T, Oowaki M, Onohara Y, Yanagi Y (2013) Deodorant performance of titanium dioxide-added acrylic/cellulose diacetate blended-fibers. Text Res J 83(8):800-812

12. JIS-P8113 (2006) Paper and board-determination of tensile properties-(in Japanese). Japanese Standard Association, Tokyo

13. Takahashi T, Kunitake T, Yokota H, Abe S, Yoshino K (2014) The effects of the traditional hand-crumpling on the performance of Manila hemp paper. Text Res J 84(6):614-625

14. JIS-P8143 (2009) Paper-Determination of stiffness-Clark stiffness tester method (in Japanese). Japanese Standard Association, Tokyo

15. JIS-P8117 (2009) Paper and board-determination of air permeance and air resistance-Gurley method- (in Japanese). Japanese Standard Association, Tokyo

16. Kunikata T, Kohno K, Ushio S, Kimura H, Ogawa S, Fukuda S (2011) Inhibitory effects of polyphenolic compounds from seed shells of the Japanese horse chestnut (Aesculus turbinate BLUME) on adherence of Helicobacter pylori to a stomach cancer cell line MKN 45. Shoyakugaku Zasshi 65(1):43-49

17. Ogawa S, Kimura H, Niimi A, Jisaka M, Katsube T, Yokota K (2009) Inhibitory effects of polyphenolic compounds from seed shells of Japanese horse chestnut (Blume) on carbohydrate-digesting enzymes. Nippon Shokuhin Kagaku Kogaku Kaishi 56(2):95-102

18. Shiratsuchi A (2012) Induction and regulation of innate immune responses by cell-wall components of Staphylococcus aureus. Biochemical 84(9):737-752

19. Toda M, Okubo S, Ikigai H, Shimamura T (1990) Antibacterial and anti-hemolysin activities of tea catechins and their structural relatives. J Jpn Soc Bacteriol 45(2):561-566

20. Yasuda H, Arakawa T (1995) Deodorizing mechanism of (-)epigallocatechin gallate against methyl mercaptan. Biosci Biotech Bioch 59(7):1232-1236

21. Griffith AA (1921) The phenomena of rupture and flow in solids. Phil Trans Roy Soc Ser A Contain Paper Math Phys Character 221:163-198 